\title{
LncRNA CASC2 Alleviates the Progression of Diabetic Nephropathy by Regulating the miR-144/SOCS2 Signalling Axis
}

\author{
Xiao-Qiang Min ${ }^{a}$ b Yan Xie $^{a}$

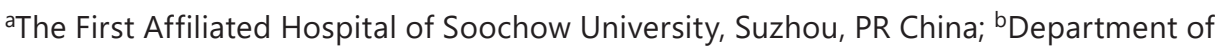 \\ Nephrology, People's Hospital of XueCheng, Zaozhuang, PR China
}

\section{Keywords}

Diabetic nephropathy · Long non-coding RNAs · microRNA · Competing endogenous RNA · Mesangial cell

\begin{abstract}
Background: Diabetic nephropathy constitutes a large proportion of end-stage kidney failure in diabetic patients. However, the underlying molecular mechanisms remain unclear. Methods: $\mathrm{Db} / \mathrm{db}$ diabetic mouse models and high glucose (HG)-induced human renal mesangial cells (HRMCs) were used as research models in vivo and in vitro. The expression of cancer susceptibility candidate 2 (CASC2) was quantified by qRT-PCR. The regulatory role of CASC2 in cell apoptosis, inflammatory factor release, and fibrosis was verified by flow cytometry, QRT-PCR, and Western blot assay, respectively. The bioinformatics prediction software DIANA and starBase v2.0 were used to predict the putative binding sites. The interactions among CASC2, miR144, and SOCS2 were explored by the luciferase assay and Western bolt assay. Results: The expression of CASC2 in diabetic mouse models and HG-induced HRMCs was lower than that in the control ( $p<0.05)$. Overexpression of CASC2 resulted in a decrease in the apoptosis rate, inflammatory factor release (TNF- $\alpha, \mathrm{IL}-6$, and IL-1 $\beta$ ), expression of cleaved caspase-3, and fibrotic proteins (fibronectin, Col-IV, and TGF- $\beta 1$ ) and an increase in Bcl-2 expression. Inhibition of CASC2 caused increased expression of miR-144. Furthermore, mechanistic investigations confirmed that activation of the miR-144/SOCS2 regulatory loop by overexpression of miR-144 reversed the in vitro effects of CASC2 on inhibiting cell apoptosis, inflammatory factor release, and fibrosis. In addition, simultaneous overexpression of miR-144 and SOCS2 further increased the inhibition of cell apoptosis, inflammatory factor release, and fibrosis by CASC2. Conclusion: CASC2 could alleviate the degree and process of apoptosis, inflammation, and fibrosis in diabetic nephropathic models by regulating the miR-144/SOCS2 axis.
\end{abstract}




\section{Introduction}

Diabetes is recognized as one of the driving causes of chronic renal failure, with an increasing number of patients advancing to end-stage renal disease [1-3]. Diabetic nephropathy (DN) is one of the foremost serious complications of diabetes, and approximately $40 \%$ of diabetic patients develop DN, which is the driving cause of end-stage renal illness [4]. Clinically, it is characterized by a dynamic increase in albuminuria and a consequent decrease in the glomerular filtration rate [5]. The major pathophysiological alterations of DN are renal cell proliferation, thickening of the glomerular basement membrane, interstitial fibrosis, and accumulation of extracellular matrix components [6]. It has been reported that DN is related to numerous pathophysiological components, such as glucose metabolic dysfunction, apoptosis, inflammation, and oxidative stress [7]. However, none of the available therapies are completely effective against DN, which implies that a better understanding of the exact mechanism underlying the pathological process of DN is vital for the improved treatment of the disease [8].

Non-coding RNA (ncRNA) is a relatively newly identified group of intermediate molecules with a broad function of regulating transcriptional and translational activities. ncRNAs are categorized into long ncRNAs (lncRNAs) and small ncRNAs. LncRNAs are transcripts with lengths surpassing 200 nucleotides that are not translated into proteins [9, 10]. Some researchers have demonstrated the relationship between lncRNAs and DN [11-17]. It is assumed that lncRNAs are involved in several pathophysiological processes in DN through interactions with microRNAs (miRNAs), including mesangial cell reactive oxidative stress and extracellular matrix accumulation $[18,19]$. Therefore, exploring the molecular elements that participate in the regulation of DN progression is of potential significance for the prevention or treatment of DN. Cancer susceptibility candidate 2 (CASC2) is a recently discovered lncRNA with crucial function in the development of all types of human neoplasms, including renal cell carcinoma [20] and diabetic chronic renal failure [15]. It has been indicated by clinical data that a low serum concentration of IncRNA CASC2 predicts the complication of chronic renal failure in type 2 diabetes patients [15]. However, the underlying mechanism of CASC2 in DN still remains unexplored.

miRNAs are 12-25-nucleotide long endogenous non-coding RNAs that cause mRNA degradation or translational repression by interacting with the $3^{\prime}$-untranslated regions $\left(3^{\prime}\right.$ UTRs) of target genes [21]. It has been reported that miRNAs are closely related to the development of DN [22]. For example, miR-485 inhibits inflammation and expansion of mesangial cells in vitro by targeting NOX5 [23]. It has also been reported that miR-218 regulates DN by targeting IKK- $\beta$ and modulating NF- $\kappa B$-mediated inflammation [24]. Moreover, miR-144 has been reported to modulate apoptosis, inflammatory factors, and fibrosis [25, 26]. Nevertheless, the mechanism of miR-144 in the pathogenesis of DN remains unknown.

In this study, human renal mesangial cells (HRMCs) and DN mouse models were used to illustrate the role of CASC2 in DN progression. CASC2 was obviously decreased in vivo and in vitro. Moreover, miR-144 was predicted to be a target of CASC2 and SOCS2 could act as a target of miR-144. Thus, we hypothesized that CASC2 might alleviate the development of DN by modulating the miR-144/SOCS2 axis, which provides a new approach and ideas for treatments of DN.

\section{Methods and Materials}

\section{Animals}

Eight-week-old male $\mathrm{db} / \mathrm{db}$ diabetic mice and their non-diabetic littermate control $\mathrm{db} / \mathrm{m}$ mice in the C57BL/KsJ background were acquired from the Model Animal Research Centre of Nanjing University (Nanjing, China). Fasting blood glucose was tested with a glucose metre 
(Sinocare, China) according to the manufacturer's instructions. Mice were placed in metabolic cages for 24-h urine collection with free access to food and water. The urine samples were centrifuged to pellet the debris, and supernatants were aliquoted and stored at $-80^{\circ} \mathrm{C}$ for later detection. Quantitative urinary albumin was measured by using a mouse ELISA kit (Sigma, St. Louis, MO, USA). The experimental procedure was approved by the Animal Care and Use Committee of The First Affiliated Hospital of Soochow University, and all experimental animals were handled in agreement with the appropriate rules and regulations.

\section{Cell Culture and High Glucose Treatment}

HRMCs were acquired from ScienCell Research Laboratories (Carlsbad, CA, USA). Cells were cultured in DMEM supplemented with $10 \%$ foetal bovine serum (FBS), $100-\mathrm{U} / \mathrm{mL}$ penicillin, and $100-\mu \mathrm{g} / \mathrm{mL}$ streptomycin at $37^{\circ} \mathrm{C}$ in a humidified atmosphere of $5 \% \mathrm{CO}_{2}$ (Invitrogen, Carlsbad, CA, USA). To mimic diabetic conditions, HRMCs were cultured under high glucose (HG; 25-mm glucose) conditions for $24 \mathrm{~h}$. Cells cultured under 5.5-mm glucose were used as controls. Cells were then harvested for subsequent assays.

\section{Histology and PAS Staining}

Samples were fixed with $10 \%$ ethyl alcohol in formalin for $10 \mathrm{~min}$ and embedded in OCT or paraffin. Seven-micron-thick sections were washed with distilled water for $10 \mathrm{~s}$. The sections were then oxidized with 1\% periodic acid solution for $10 \mathrm{~min}$. The sections were rinsed with distilled water for $10 \mathrm{~s}$ and blotted dry with filter paper, followed by air drying at room temperature. The sections were immersed in the Schiff reagent for 30 min (ABRIS+, stored at $4^{\circ} \mathrm{C}$ ), carefully washed with distilled water for $5 \mathrm{~min}$, counterstained with haematoxylin for $5 \mathrm{~min}$, and rinsed with tap water.

\section{Cell Transfection and Plasmid Construction}

NC mimics, miR-144 mimics, NC inhibitor, and miR-144 inhibitor were acquired from RiboBio (Guangzhou, China). The sequences were as follows: mimics NC forward, $5^{\prime}$-UUCUCCGAACGUGUCACGUTT- $3^{\prime}$, and reverse, $5^{\prime}$-ACGUGACACGUUCGGAGAATT- $3^{\prime \prime}$; miR-144 mimics forward, $5^{\prime}$-UACAGUAUAGAUGAUGUACU- $3^{\prime}$, and reverse, $5^{\prime}$-UACAUCAUCUAUACUGUAUU- ${ }^{\prime \prime}$; NC inhibitor 5' $5^{\prime}$-CAGUACUUUUGUGUAGUACAAA-3'; and miR-144 inhibitor 5'-UACAGUAUAGAUGAUGUACU- $3^{\prime \prime}$. Full-length HULC and SOCS2 sequences were amplified and ligated into the pcDNA3.1 plasmid (Gene Pharma, Shanghai, China), and the recombinant plasmids were named pcDNA3.1-HULC and pcDNA3.1-SOCS2, respectively. The cells were transfected with miR-144 mimics, NC mimics, miR-144 inhibitor, or NC inhibitor (50 nm) using Lipofectamine 2000 reagent (Invitrogen, Carlsbad, CA, USA), according to the manufacturer's protocol. The cells were collected for further use after $48 \mathrm{~h}$ of transfection.

\section{Quantitative Real-Time PCR}

Total RNA was isolated from cells by using TRIzol reagent (Invitrogen Life Technologies, Waltham, MA, USA) following the manufacturer's protocol. RNA concentration was measured by using a NanoPhotometer (Implen GmbH, Germany). Reverse transcription involved the use of a cDNA synthesis kit (Takara, China). Gene expression was quantitatively analyzed by using SYBR Green with the 7500 Real-Time PCR system (Takara, China). The expression of each gene was normalized to that of GAPDH and analyzed by the $2^{-\triangle \triangle C T}$ method. Primer sequences are listed in Table 1. All experiments were performed in triplicate.

Flow Cytometry Analysis

For flow cytometry analysis, a total of $3 \times 10^{5}$ cells were cultured in 6-well plates for $24 \mathrm{~h}$. The cells were harvested using trypsin, washed twice with PBS, and subsequently

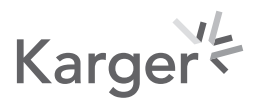


Table 1. Primer sequences for qRT-PCR

\begin{tabular}{lll}
\hline Gene & Forward sequence & Reverse sequence \\
\hline CASC2 & 5'-ATTGGACGGTGTTTCCGTGT-3' & 5'-ACCACCACTGACTGTCCTGT-3' \\
Hsa-miR-144-3p & 5'-GGCCGGCGTACAGTATAGATGA-3' & 5'-GTGCAGGGTCCGAGGT-3' \\
SOCS2 & 5'-GTTCGGACTCTCTCTTCCCC-3' & 5'-ATCAAATCCTGGGCCACACA-3' \\
TNF- $\alpha$ & 5'-CTGGGCAGGTCTACTTTGGG-3' & 5'-CTGGAGGCCCCAGTTTGAAT-3' \\
IL-6 & 5'-CCAGCTATGAACTCCTTCTC-3' & 5'-GCTTGTTCCTCACATCTCTC-3' \\
IL-1 $\beta$ & 5'-AAGCTGATGGCCCTAAACAG-3' & 5'-AAGCTGATGGCCCTAAACAG-3' \\
U6 & 5'-CTCGCTTCGGCAGCACA-3' & 5'-AACGCTTCACGAATTTGCGT-3' \\
GAPDH & 5'-CCAGGTGGTCTCCTCTGA-3' & 5'-GCTGTAGCCAAATCGTTGT-3' \\
\hline
\end{tabular}

CASC2, cancer susceptibility candidate 2 .

subjected to an apoptosis assay with an Annexin V-FITC/PI Apoptosis Detection Kit (BD Pharmingen; BD Biosciences) according to the manufacturer's protocol. Finally, apoptotic cells were analyzed by flow cytometry with FACSD (BD Bioscience, San Jose, CA, USA). The data were further analyzed by using FlowJo software (Treestar, San Carlos, CA, USA).

\section{Western Blot}

Cells were harvested and lysed in RIPA buffer (Beyotime, Nanjing, China). SDS-PAGE gels $(12 \%)$ were used to separate proteins, which were transferred to a methanol-activated PVDF membrane (GE Healthcare, Chicago, IL, USA). After blocking for $1 \mathrm{~h}$ in PBST containing 5\% BSA, the membrane was probed with the following primary antibodies: anti-Bcl-2 (ab32124), anti-cleaved caspase-3 (ab2302), anti-fibronectin (ab2413), anti-collagen IV (ab6586), antiTGF- $\beta 1$ (ab92486), and anti-GAPDH (ab181602; all from Abcam, UK) overnight at $4^{\circ} \mathrm{C}$. After $2 \mathrm{~h}$ of incubation with goat-anti-rabbit horseradish peroxidase-conjugated secondary antibody (Santa Cruz Biotechnology, Santa Cruz, CA, USA), the bands were analyzed by using an ECL detection kit (Pierce Biotechnology, Waltham, MA, USA).

\section{Dual-Luciferase Reporter Assay}

The assay was carried out as described before [27]. Wild-type and mutant reporter plasmids of CASC2 (CASC2-WT-luc and CASC2-MUT-luc) and SOCS2 (SOCS2-WT-luc and SOCS2-MUT-luc) containing wild-type or mutant miR-144 mimics or mimics NC binding sites were synthesized by Gene Pharma (Shanghai, China). The synthesized reporter plasmids were co-transfected with miR-144 mimics or NC mimics by Lipofectamine 2000 (Invitrogen) when the cells reached $70 \%$ confluence. The luciferase activity was analyzed by the DualLuciferase Reporter Assay System (Promega, Madison, WI, USA) after 48 h.

\section{Statistical Analysis}

All data are presented as the means $\pm S D$, and each assay was repeated in triplicate. Statistical analyses were performed using GraphPad Prism 5.0 software (GraphPad, La Jolla, CA, USA). Significant differences were calculated by one-way ANOVA followed by Tukey's post hoc test for multiple comparisons or Student's $t$ test. $p<0.05$ was considered a statistically significant difference.

\section{Karger's}




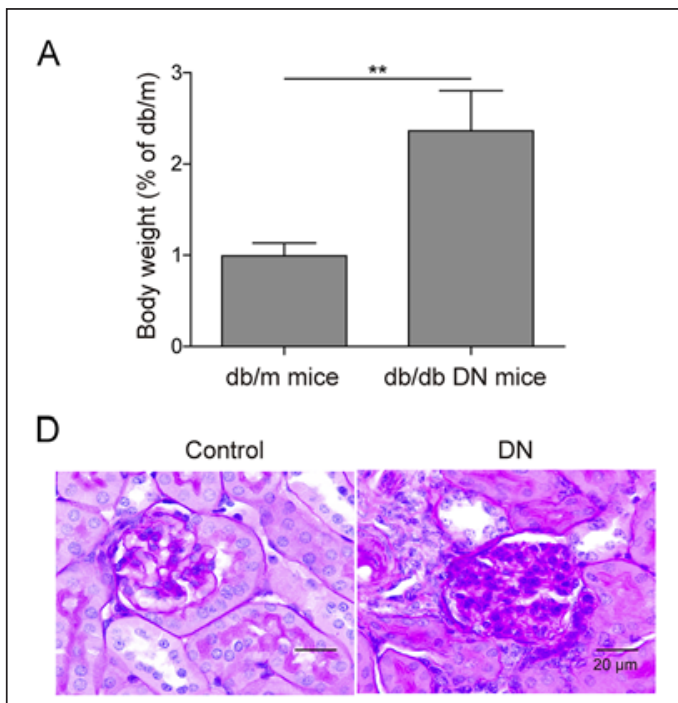

B

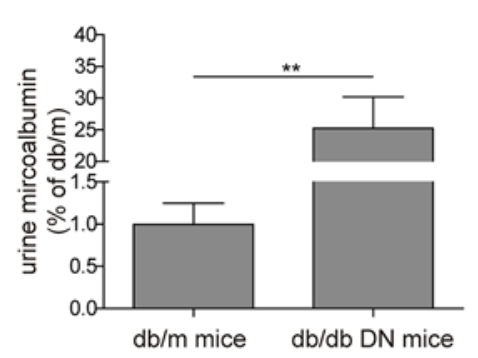

E

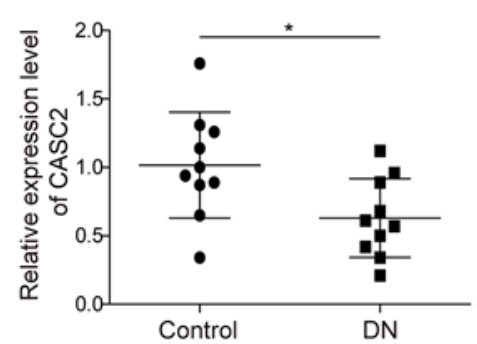

C

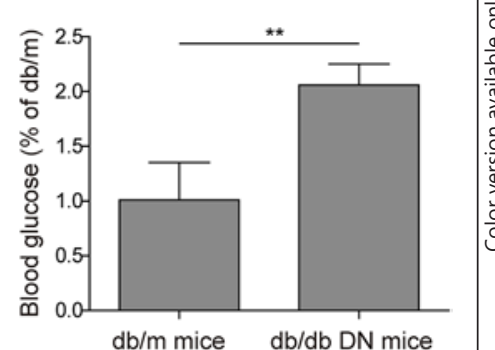

$\mathrm{F}$

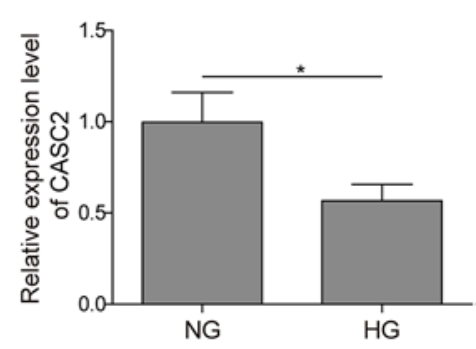

Fig. 1. CASC2 is downregulated in DN mice and HG-induced HRMCs. After DN modelling, the body weights of normal $\mathrm{db} / \mathrm{m}$ mice and $\mathrm{db} / \mathrm{db}$ diabetic mice were recorded $(\mathbf{A})$; urine microalbumin levels were detected by an ELISA kit (B); blood glucose levels were tested by using a glucose metre (C); and renal injury was evaluated by PAS staining (D). E, F HRMCs were cultured in the medium with $\mathrm{HG}$ for $24 \mathrm{~h}$, and the relative expression of CASC2 in diabetic mice and HG-induced HRMCs was measured by qRT-PCR. Data are shown as the mean $\pm \mathrm{SD}, n=6$; ${ }^{*} p<0.05$ and ${ }^{* *} p<0.01$. CASC2, cancer susceptibility candidate 2 ; DN, diabetic nephropathy; $\mathrm{HG}$, high glucose; HRMCs, human renal mesangial cells.

\section{Results}

\section{CASC2 Is Downregulated in DN Mice and HG-Induced HRMCs}

$\mathrm{Db} / \mathrm{db}$ diabetic mice and HG-induced HRMCs were used to analyze CASC2 expression in vivo and in vitro. Compared to normal $\mathrm{db} / \mathrm{m}$ mice, $\mathrm{db} / \mathrm{db}$ diabetic mice demonstrated larger body weight $(p<0.01)$, higher levels of blood glucose $(p<0.01)$, and increased urine microalbumin $(p<0.01)$, as shown in Figure 1A-C. Subsequently, PAS staining was used to assess renal extracellular matrix accumulation. As shown in Figure 1D, significant extracellular matrix accumulation was observed in $\mathrm{db} / \mathrm{db}$ diabetic mice compared with normal $\mathrm{db} / \mathrm{m}$ mice. The relative expression of CASC2 in DN mice and HRMCs was assessed by qRT-PCR. As expected, CASC2 had lower expression in DN mice and HG-induced HRMCs than in control mice $(p<0.05$; Fig. 1E, F). These data suggest that CASC2 could play an important role in regulating the process of DN.

CASC2 Suppresses the Apoptosis, Inflammatory Factor Release, and Fibrosis of HRMCs

To detect the effect of CASC2 on HG-induced HRMCs in different pathological processes, a CASC2 overexpression vector (pcDNA3.1-CASC2) was transfected into HRMCs. The expression level of CASC2 was downregulated after HG induction $(p<0.05)$, while the overexpression of CASC2 significantly upregulated CASC2 expression $(p<0.01$, Fig. 2A). Subsequently, flow cytometry was used to detect the apoptosis rate. The results showed that HG significantly promoted apoptosis $(p<0.01)$ and overexpression of CASC2 clearly attenuated HG-induced apoptosis ( $p<0.05$; Fig. 2B). In addition, Western blot analysis suggested that 


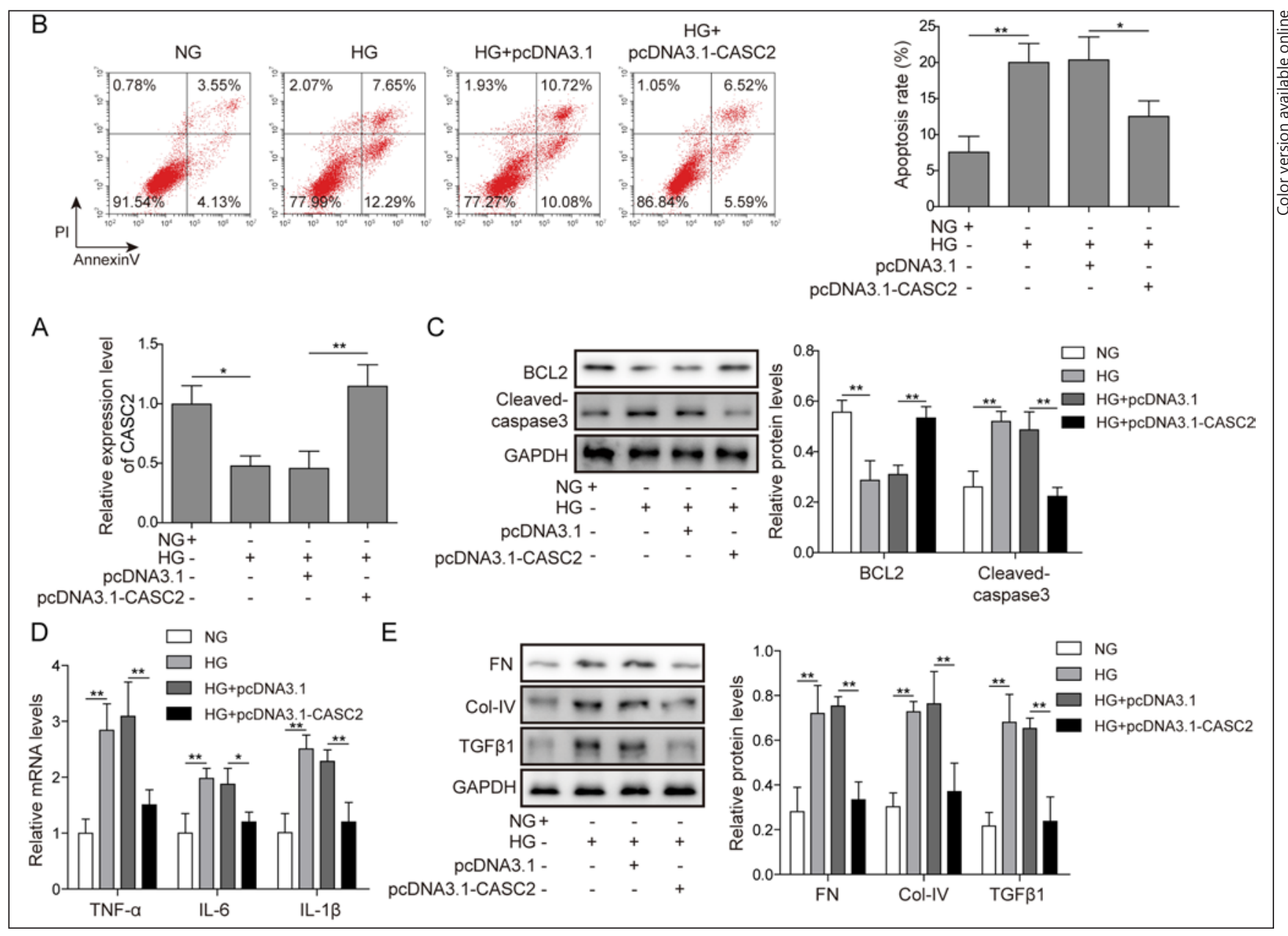

Fig. 2. CASC2 suppresses the apoptosis, inflammatory factor release, and fibrosis of HRMCs. HG-induced HRMCs were transfected with the CASC2 overexpression vector (pcDNA-CASC2) or empty vector plasmid (pcDNA-3.1). The expression levels of CASC2 were tested by qRT-PCR (A); cell apoptosis was analyzed by flow cytometry (B); the expression of Bcl-2 and cleaved caspase-3 was detected by Western blot (C); the expression of TNF- $\alpha$, IL-6, and IL-1 $\beta$ was determined by qRT-PCR (D); and the expression levels of FN, Col-IV, and TGF- $\beta 1$ were analyzed by Western blot (E). Data are shown as the mean \pm SD, $n=3 ;{ }^{*} p<0.05$ and ${ }^{* *} p<$ 0.01 . CASC2, cancer susceptibility candidate 2; DN, diabetic nephropathy; HG, high glucose; HRMCs, human renal mesangial cells.

Bcl-2 was significantly downregulated and cleaved caspase-3 was markedly increased after HG induction, while overexpression of CASC2 significantly promoted Bcl-2 and suppressed cleaved caspase-3 ( $p<0.01$; Fig. 2C). As shown in Figure 2D, qRT-PCR was performed to assay the relative expression of inflammatory factors, and the data suggested that after HG treatment, the expression levels of TNF- $\alpha$, IL- 6 , and IL- $1 \beta$ were significantly promoted $(p<0.01)$. However, overexpression of CASC2 remarkably downregulated the expression levels of TNF- $\alpha$, IL-6, and IL-1 $\beta$. Western blotting also revealed that HG treatment induced increased protein levels of FN, Col-IV, and TGF- $\beta$ in HRMCs, while overexpression of CASC2 inhibited the effects of HG treatment on these proteins ( $p<0.01$; Fig. 2E). Taken together, the results demonstrated that CASC2 suppressed the apoptosis, inflammatory factor release, and fibrosis of HRMCs. 


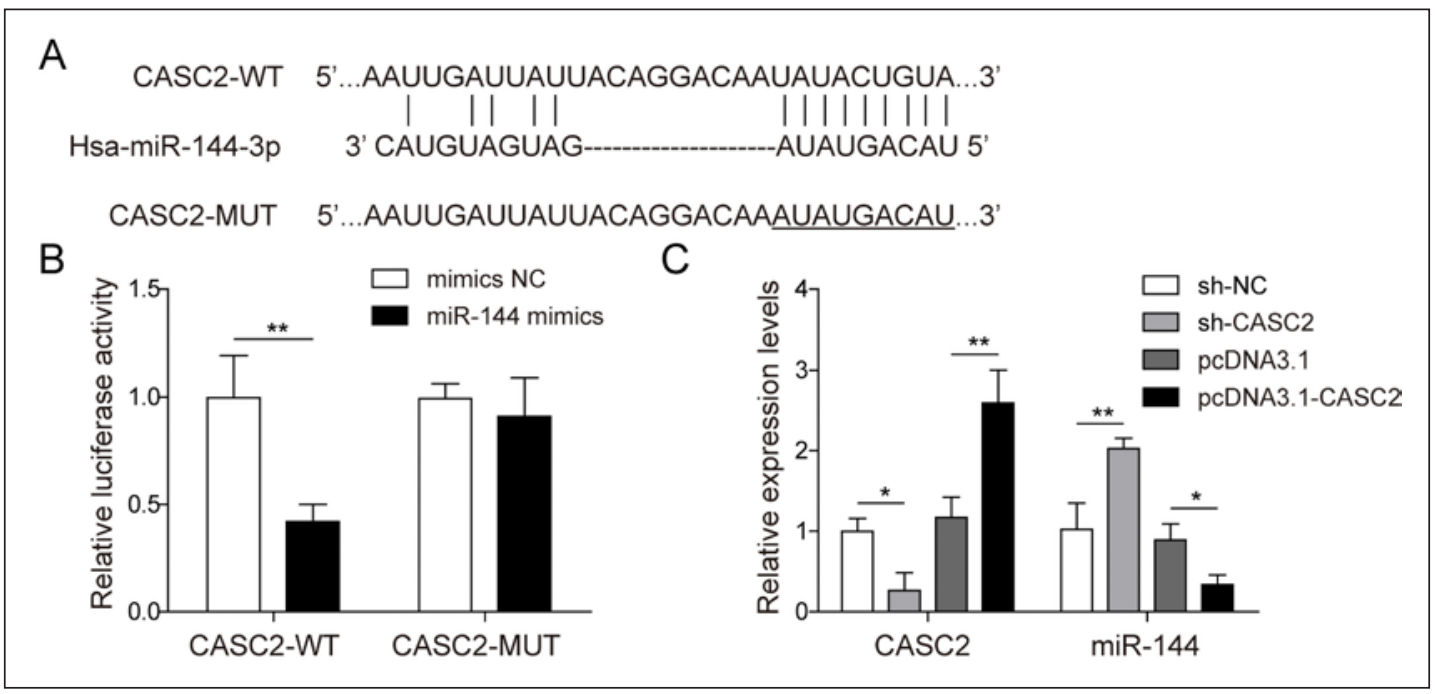

Fig. 3. CASC2 suppressed the expression of miR-144. The prediction of binding sites between CASC2 and miR-144 was performed using DIANA TOOLS. A The miR-144 mimics and luciferase reporter plasmids with wild-type or mutant CASC2 3'-UTR were co-transfected into HRMCs. B A dual-luciferase reporter assay was performed to explore the direct binding relationship between CASC2 and miR-144. C HRMCs were transfected with sh-CASC2, sh-NC, CASC2 overexpression vector (pcDNA3.1-CASC2), or empty vector plasmid (pcDNA-3.1), and then qRT-PCR was performed to evaluate the relative expression levels of CASC2 and miR144. Data are shown as the mean $\pm \mathrm{SD}, n=3 ;^{*} p<0.05$ and ${ }^{* *} p<0.01$. CASC 2 , cancer susceptibility candidate 2; HRMCs, human renal mesangial cells; 3'-UTR, 3'-untranslated regions; sh-CASC2, CASC2-specific shRNA; sh-NC, negative control shRNA.

\section{CASC2 Suppressed the Expression of miR-144}

To uncover the downstream targets of CASC2, we used DIANA software to predict the downstream-targeted miRNAs that could bind and interact with CASC2. As shown in Figure 3A, miR-144 was selected as the target downstream miRNA for further study. Next, a dual-luciferase reporter assay revealed that the luciferase activity of cells co-transfected with miR-144 mimics and wild-type CASC2 vector was notably decreased compared to that of cells co-transfected with miR-144 mimics and mutant CASC2 vector ( $p<0.01$; Fig. 3B). Further qRT-PCR detection showed that knockdown of CASC2 was directly related to upregulated miR-144 expression, whereas overexpression of CASC2 obviously downregulated miR-144 expression (Fig. 3C). Together, these results demonstrated that CASC2 directly interacts with miR-144.

CASC2 Acted as a ceRNA and Upregulated the Expression of SOCS2 by Sponging miR-144

To discover the potential target genes mediated by miR-144 in HRMCs, we identified SOCS2 as one of the target genes of miR-144 through starBase software (Fig. 4A). Luciferase activity assays provided further evidence that SOCS2 could be a downstream target of miR-144. The results demonstrated that decreased luciferase activity was observed in cells co-transfected with miR-144 mimics and SOCS2-WT, compared to cells co-transfected with SOCS2-WT and mimics NC. There was no significant change in luciferase activity in cells co-transfected with SOCS2-MUT vector ( $p<0.05$; Fig. 4B). Moreover, qRT-PCR and Western blotting showed that overexpression of miR-144 decreased the mRNA and protein expression of SOCS2, while knockdown of miR-144 increased the expression of SOCS2 at both the mRNA and protein levels (Fig. 4C, D), suggesting that miR-144 negatively regulated the expression of SOCS2. Furthermore, overexpression of CASC2 caused a significant increase in the expression of SOCS2, whereas miR-144 mimics resulted in reduced SOCS2 expression at both 


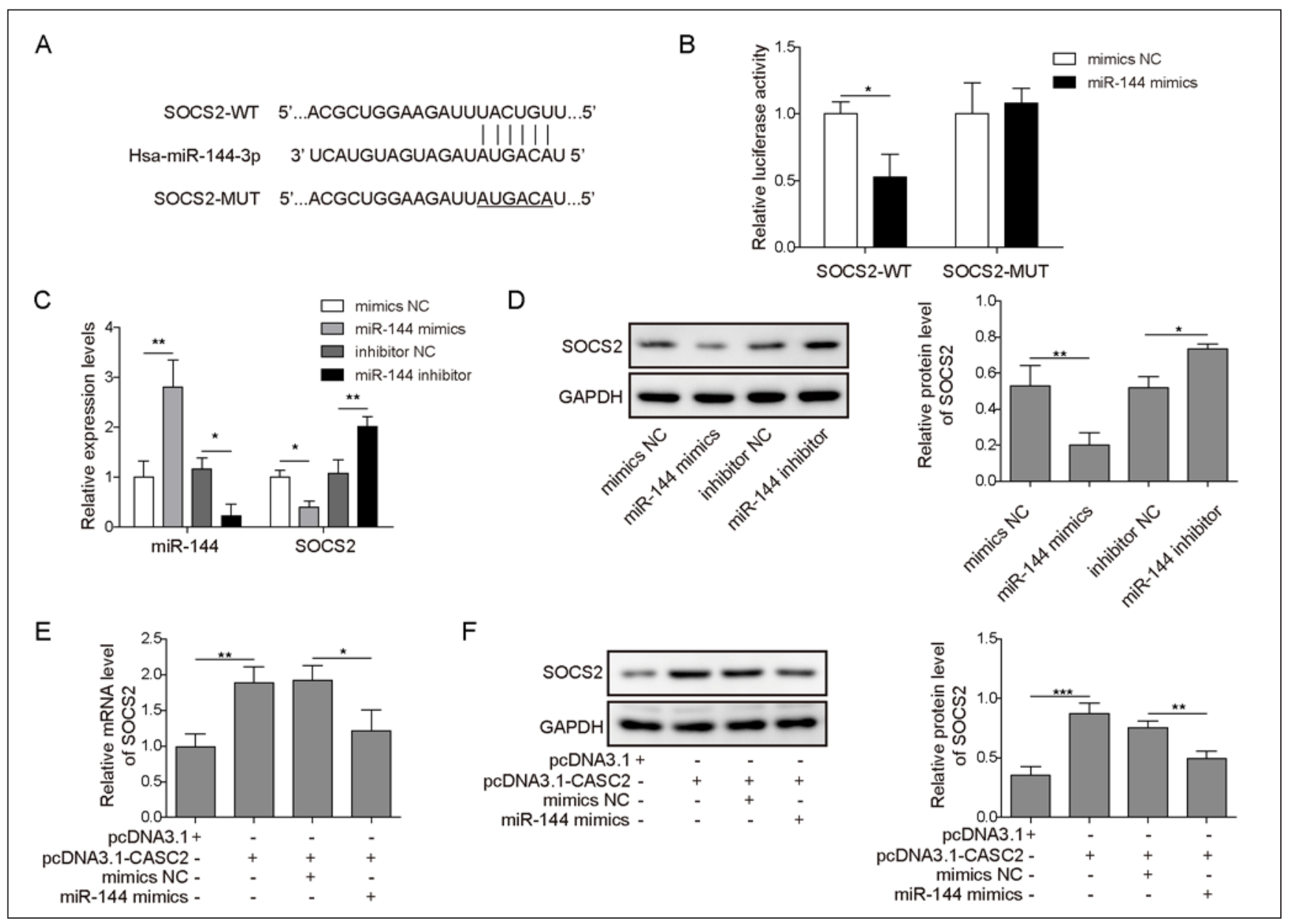

Fig. 4. CASC2 acted as a ceRNA and upregulated the expression of SOCS2 by sponging miR-144. A The potential binding sites between miR-144 and SOCS2 were predicted by starBase software. The miR-144 mimics and luciferase reporter plasmids with wild-type or mutant SOCS2 3'-UTR were co-transfected into HRMCs. B A dual-luciferase reporter assay was performed to verify the direct binding relationship between miR-144 and SOCS2. C HRMCs were transfected with mimics NC, miR-144 mimics, inhibitor NC, or miR-144 inhibitor, and then qRT-PCR was performed to evaluate the relative expression levels of miR-144 and SOCS2. D The expression level of SOCS2 was analyzed by Western blot. E HRMCs were transfected with pcDNA3.1, pcDNA3.1-CASC2, pcDNA3.1-CASC2+ mimics NC, and pcDNA3.1-CASC2+miR-144 mimics, and then qRT-PCR was performed to evaluate the relative mRNA expression levels of SOCS2. F The protein expression level of SOCS2 was analyzed by Western blot. Data are shown as the mean $\pm \mathrm{SD}, n=3$; ${ }^{*} p<0.05$, ${ }^{* *} p<0.01$, and ${ }^{* * *} p<0.001$. CASC2, cancer susceptibility candidate 2; HRMCs, human renal mesangial cells; 3 '-UTR, 3'-untranslated regions; ceRNA, competing endogenous RNA.

the mRNA and protein levels (Fig. 4E, F). Collectively, these data strongly suggest that CASC2 most likely acts as a competing endogenous RNA (ceRNA) by sponging cellular miR-144, thereby regulating SOCS2 expression.

\section{miR-144 Reversed the CASC2-Mediated Apoptosis, Inflammatory Factor Release, and}

Fibrosis of HRMCS

To investigate the role of miR-144 in CASC2-related pathological processes of HRMCs, both CASC2 and miR-144 were overexpressed in HG-induced HRMCs and relevant pathological markers were detected. As shown in Figure 5A, overexpression of CASC2 reduced the 


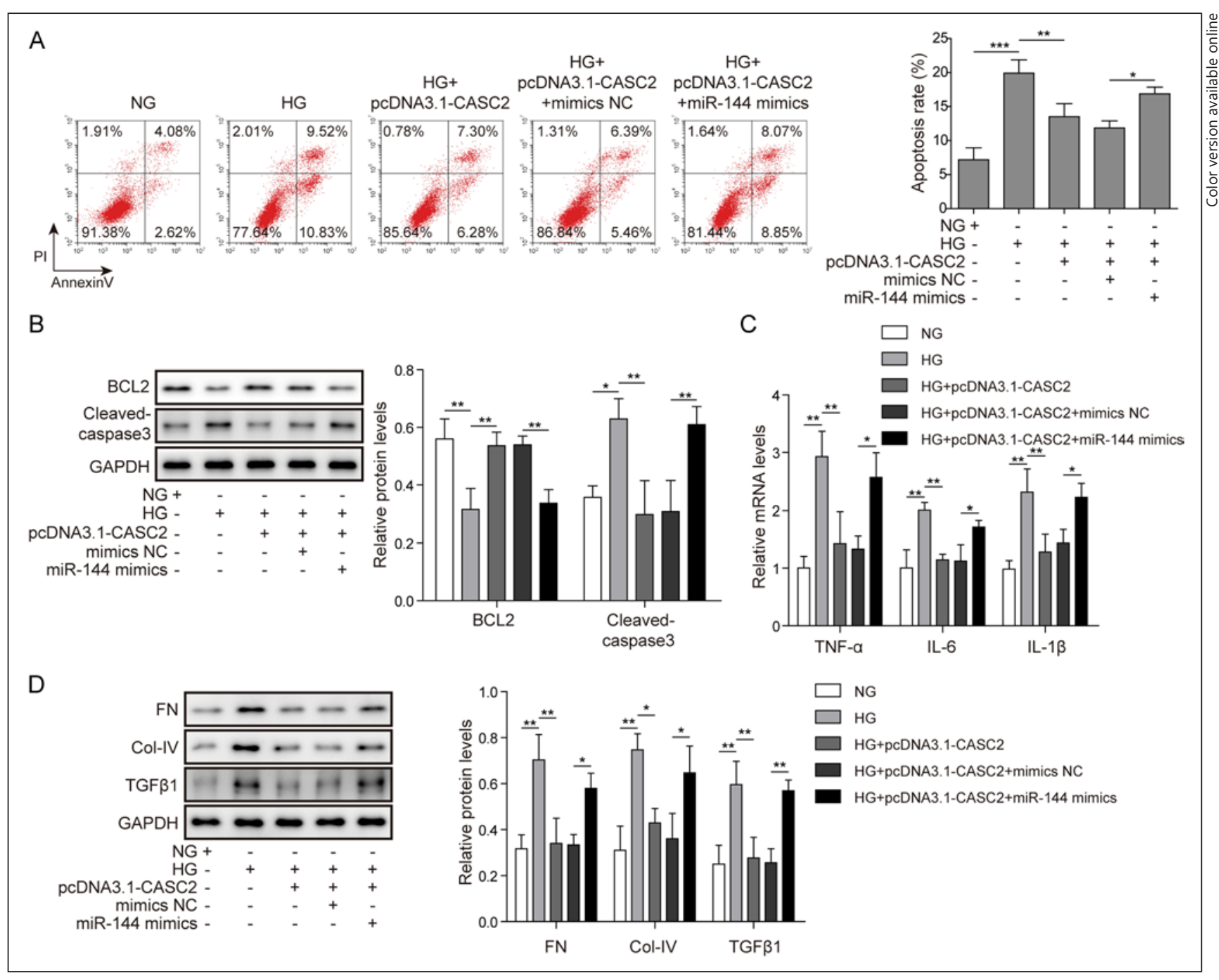

Fig. 5. miR-144 reversed the CASC2-mediated apoptosis, inflammatory factor release, and fibrosis of HRMCs. HG-induced HRMCs were co-transfected with the CASC2 overexpression vector (pcDNA3.1-CASC2) and mimics NC or miR-144 mimics. Cell apoptosis was analyzed by flow cytometry (A); the expression of Bcl-2 and cleaved caspase-3 was detected by Western blot (B); the expression of TNF- $\alpha$, IL-6, and IL-1 $\beta$ was determined by qRT-PCR (C); and the expression levels of FN, Col-IV, and TGF- $\beta 1$ were analyzed by Western blot (D). Data are shown as the mean $\pm \mathrm{SD}, n=3$; ${ }^{*} p<0.05,{ }^{* *} p<0.01$, and ${ }^{* * *} p<0.001$. CASC2, cancer susceptibility candidate 2; DN, diabetic nephropathy; HG, high glucose; HRMCs, human renal mesangial cells.

apoptosis rate induced by HG $(p<0.01)$. However, overexpression of miR-144 reversed that effect $(p<0.05)$. Similarly, overexpression of CASC2 could upregulate Bcl-2 and downregulate cleaved caspase-3 in HG-induced HRMCs ( $p<0.01)$, while overexpression of miR-144 reversed the previous effect $(p<0.01$; Fig. 5B). Overexpression of CASC2 dramatically decreased the expression of the inflammatory factors TNF- $\alpha$, IL- 6 , and IL- $1 \beta$ in HG-induced HRMCs $(p<0.01)$. A similar effect was also observed on the expression of fibrotic proteins. The results showed that the overexpression of CASC2 suppressed the expression of FN, Col-IV, and TGF- $\beta 1$, but the influence of CASC2 on inflammatory factors and fibrotic proteins was also reversed by the overexpression of miR-144 (Fig. 5C, D). Overall, the results indicated that miR-144 reversed the CASC2-mediated apoptosis, inflammatory factor release, and fibrosis of HRMCs. 


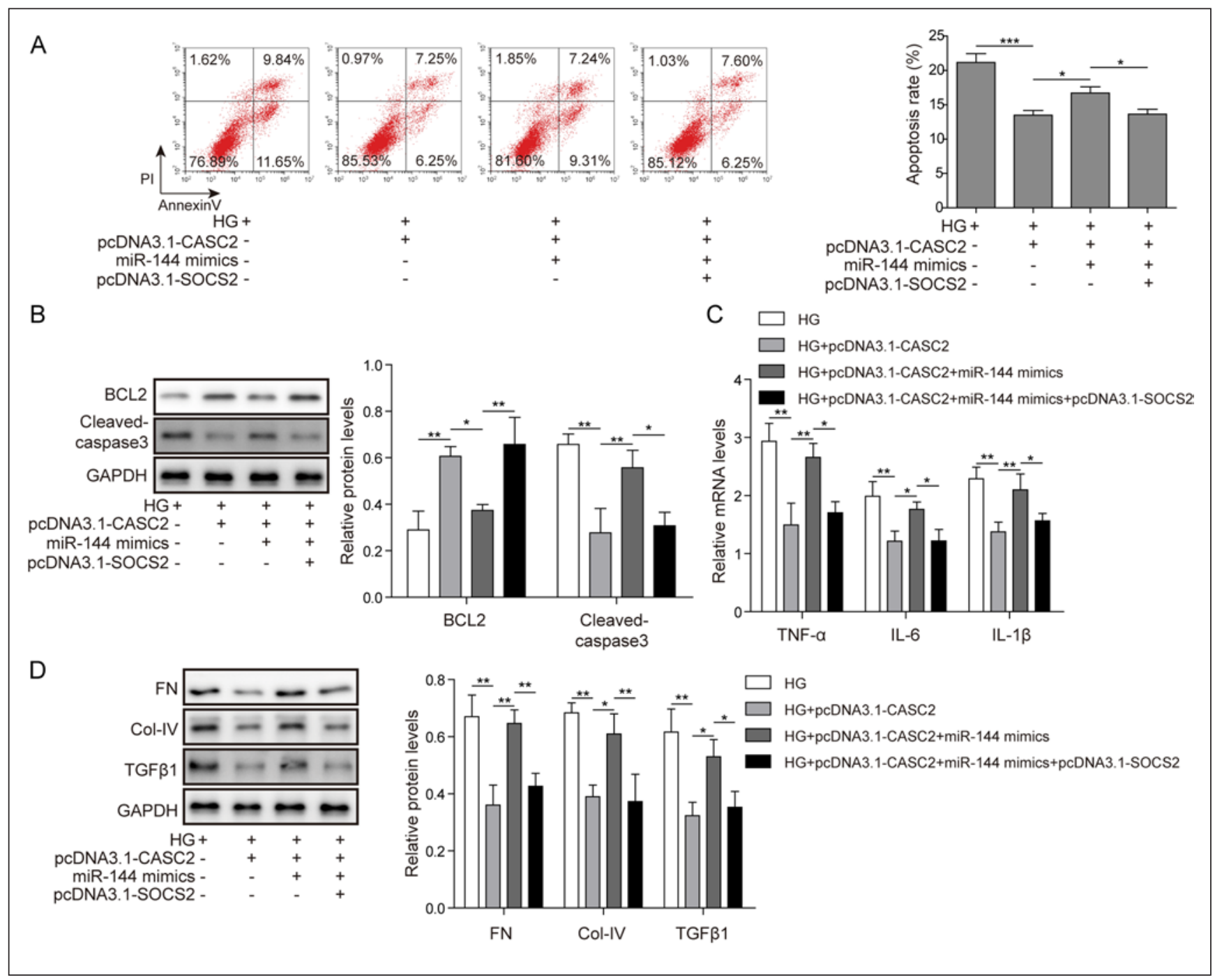

Fig. 6. CASC2 regulates the HG-induced pathological process of HRMCs via the miR-144/SOCS2 axis. HG-induced HRMCs were transfected with the CASC2 overexpression vector (pcDNA3.1-CASC2), CASC2 and miR144 mimics, or CASC2 and miR-144 mimics together with the SOCS2 overexpression vector (pcDNA3.1SOCS2). Cell apoptosis was analyzed by flow cytometry (A); the expression of Bcl-2 and cleaved caspase-3 was detected by Western blot (B); the expression of TNF- $\alpha$, IL-6, and IL-1 $\beta$ was determined by qRT-PCR (C); and the expression levels of FN, Col-IV, and TGF- $\beta 1$ were analyzed by Western blot (D). Data are shown as the mean $\pm \mathrm{SD}, n=3{ }^{*} p<0.05$, ${ }^{* *} p<0.01$, and ${ }^{* * *} p<0.001$. CASC2, cancer susceptibility candidate 2; DN, diabetic nephropathy; HG, high glucose; HRMCs, human renal mesangial cells.

CASC2 Regulated HG-Induced Pathological Process of HRMCS via the miR-144/SOCS2 Axis

Based on the aforementioned results, we tested whether CASC2 mediates the HG-induced pathological process via the miR-144/SOCS2 axis by rescue experiments. Overexpression of SOCS2 was achieved by transfecting HRMCs with the pcDNA3.1-SOCS2 vector. The results demonstrated that overexpression of CASC2 decreased the apoptosis rate, upregulated the expression of Bcl-2 and downregulated that of cleaved caspase-3, reduced inflammatory factors, and decreased the protein levels of FN, Col-IV, and TGF- $\beta 1$. Overexpression of miR-144 reversed the above effects. Furthermore, co-overexpression of CASC2, miR-144, and SOCS2 caused a remarkable decrease in apoptosis, the expression of cleaved caspase-3, inflam- 
matory factors, and fibrotic proteins, but recovered the expression of Bcl-2 when compared with that of cells overexpressing CASC2 and miR-144 (Fig. 6A-D). Accordingly, the rescue experiment data indicated that CASC2 alleviated DN-related pathological processes by regulating the miR-144/SOCS2 axis.

\section{Discussion}

A number of publications have reported that lncRNAs are directly related to the pathophysiological development of DN [28, 29]. For example, knockdown of CDKN2B-AS1 suppressed mesangial cell proliferation and ECM accumulation through the miR-424-5p/ HMGA2 axis [30]. A previous study discovered the correlation between CASC2 and the diagnosis and prognosis of patients suffering from DN [15]. However, none of the downstream genes or other molecular interactions have been revealed regarding the role of CASC2 in the development of DN. In our study, diabetic mice and HG-treated HRMCs were used to establish diabetic animal and cell models. We found that CASC2 was markedly downregulated in vivo and in vitro (Fig. 1). Apoptosis, inflammation, and fibrosis of mesangial cells are well recognized as 3 main pathophysiological processes of DN [1, 8, 31-33]. In the following cellular experiments, we found that overexpression of CASC2 dramatically suppressed the apoptosis, inflammatory factor release, and fibrosis in HG-induced HRMCs (Fig. 2), demonstrating the potential of CASC2 as a therapeutic target for DN intervention. Therefore, we next explored the underlying mechanism.

Related research has shown that IncRNAs control gene expression at different levels, which include epigenetic transcriptional and post-transcriptional changes to mediate chromosomal adjustment, transcriptional repression, or transcriptional activation [10, 34]. Recently, ceRNAs, which indicate IncRNAs that can manipulate other transcripts by competing for binding to microRNAs, have attracted increasing attention $[35,36]$. The IncRNA LEGLTBC has been discovered to act as a ceRNA to antagonize the impacts of miR-34a on the downregulation of SIRT1 in glucolipotoxicity-induced INS-1 beta cell oxidative stress and apoptosis [37]. As a ceRNA, LINC00511 was also found to facilitate cell malignant behaviours and relate to hepatocellular carcinoma patient prognosis by interfering with the miR-195/EYA1 axis [38]. Similarly, we found that there was a conserved binding site of miR-144 on CASC2 by bioinformatics analyses. Therefore, we hypothesized that CASC2 could affect miR-144 via a ceRNA mechanism. As an encouraging result, we further demonstrated that CASC2 directly interacts with miR-144 to reduce its expression, as demonstrated by the dual-luciferase assay and qRT-PCR (Fig. 3), suggesting CASC2 acts as a miRNA sponge that binds to and regulates miR-144 expression.

Increasing and emerging evidence has illustrated that miRNAs are vital regulators in the development of DN by binding to the 3 '-UTR of the target gene, which causes mRNA cleavage or translational repression $[23,39]$. To explore the target genes of miR-144, starBase v2.0 software was used and predicted SOCS2 as one of the targets of miR-144. SOCS2, which is regarded as an irreplaceable regulator of inflammatory responses, has been published to strongly affect the pathogenesis of DN [40]. Previous reports clarified that overexpression of SOCS2 reduced the expression of inflammatory cytokines and fibrosis-related proteins in DN rats [41]. Similarly, our dual-luciferase assays confirmed the direct interaction between miR-144 and SOCS2, and SOCS2 was negatively regulated by miR-144 (Fig. 5). Furthermore, rescue experiments showed that CASC2 alleviated the HG-induced cell apoptosis, inflammatory factor release, and fibrosis in HRMCs via regulation of the miR-144/SOCS2 signalling axis (Fig. 6).

Although this study revealed a novel downstream regulatory mechanism of DN involving CASC2 in vitro, there is still much to be implemented. As is the body is more complicated and

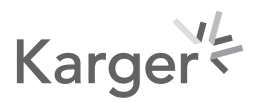


has more uncertainty than cell models, future animal model studies are needed to further verify the novel signalling pathway and demonstrate its potential as a therapeutic target.

In conclusion, our study demonstrates that CASC2 acts as a ceRNA to regulate SOCS2 expression by competitively sponging miR-144, thereby regulating the progression of DN. Therefore, our findings might provide a new avenue and resolution of genetic therapies for DN.

\section{Statement of Ethics}

The exploratory plan was endorsed by the Creature Care and Utilize Committee of The First Affiliated Hospital of Soochow University, and all experimental animals were kept up in agreement with the rules.

\section{Conflict of Interest Statement}

The authors declare that they have no conflicts of interest.

\section{Funding Sources}

The authors did not receive any funding.

\section{Author Contributions}

X.Q.M.: conception or design of the work; Y.X.: acquisition or analysis or interpretation of data for the work; X.Q.M. and Y.X.: drafting the work or revising it critically for important intellectual content, final approval of the version to be published, and agree to be accountable for all aspects of the work.

\section{References}

1 Jha JC, Banal C, Chow BS, Cooper ME, Jandeleit-Dahm K. Diabetes and kidney disease: role of oxidative stress. Antioxid Redox Signal. 2016;25(12):657-84.

2 Zimmet P, Alberti KG, Magliano DJ, Bennett PH. Diabetes mellitus statistics on prevalence and mortality: facts and fallacies. Nat Rev Endocrinol. 2016;12(10):616-22.

3 Forbes JM, Cooper ME. Mechanisms of diabetic complications. Physiol Rev. 2013;93(1):137-88.

4 Chu C, Li D, Zhang S, Ikejima T, Jia Y, Wang D, et al. Role of silibinin in the management of diabetes mellitus and its complications. Arch Pharm Res. 2018;41(8):785-96.

5 Sheen YJ, Sheu WH. Risks of rapid decline renal function in patients with type 2 diabetes. World J Diabetes. 2014;5(6):835-46.

6 Yiu WH, Lin M, Tang SC. Toll-like receptor activation: from renal inflammation to fibrosis. Kidney Int Suppl. 2011;4(1):20-5..2014

7 An Y, Xu F, Le W, Ge Y, Zhou M, Chen H, et al. Renal histologic changes and the outcome in patients with diabetic nephropathy. Nephrol Dial Transplant. 2015;30(2):257-66.

8 Kato M, Natarajan R. Diabetic nephropathy: emerging epigenetic mechanisms. Nat Rev Nephrol. 2014;10(9): 517-30.

9 Flynn RA, Chang HY. Long noncoding RNAs in cell-fate programming and reprogramming. Cell Stem Cell. 2014; 14(6):752-61.

10 Orom UA, Shiekhattar R. Long noncoding RNAs usher in a new era in the biology of enhancers. Cell. 2013; 154(6):1190-3.

11 MA-Ohoo K, Wang M, Chen Z, Bhatt K, Oh HJ, Lanting L, et al. An endoplasmic reticulum stress-regulated lncRNA hosting a microRNA megacluster induces early features of diabetic nephropathyNat Commun2016 Sep 30712864 
12 Zhou Q, Chung A, CHuang XR, Dong Y, Yu X, Lan HY, et al. Identification of novel long noncoding RNAs associated with TGF-beta/Smad3-mediated renal inflammation and fibrosis by RNA sequencingAm J Pathol2014 Feb184240917

13 Long J, Badal SS, Ye Z, Wang Y, Ayanga BA, Galvan DL, et al. Long noncoding RNA Tug1 regulates mitochondrial bioenergetics in diabetic nephropathyl Clin Invest2016 Nov 112611420518

14 Liu JY, Yao J, Li XM, Song YC, Wang XQ, Li YJ, et al. Pathogenic role of IncRNA-MALAT1 in endothelial cell dysfunction in diabetes mellitusCell Death Dis2014 Oct 30510e1506

15 Yan Y, Kang Y, Li D, Yu K, Xiao T, Wang Q, et al. Microstructure, mechanical properties and corrosion behavior of porous Mg-6 wt.\% Zn Scaffolds for bone tissue engineering. J Mater Eng Perform. 2018;27:970-84.

16 Li Y, Xu K, Xu K, Chen S, Cao Y, Zhan H. Roles of identified long noncoding RNA in diabetic nephropathy. J Diabetes Res. 2019;2019:5383010.

17 Kato M, Wang M, Chen Z, Bhatt K, Oh HJ, Lanting L, et al. An endoplasmic reticulum stress-regulated lncRNA hosting a microRNA megacluster induces early features of diabetic nephropathy. Nat Commun. 2016;7:12864.

18 Zhao J, Chen L-j, Yu K, Chen C, Dai Y-l, Qiao X-y, et al. Effects of chitosan coating on biocompatibility of Mg-6\%Zn-10\%Ca3(P04)2 implant. Trans Nonferrous Met Soc China. 2015;25:824-31.

19 Yi H, Peng R, Zhang LY, Sun Y, Peng HM, Liu HD, et al. LincRNA-Gm4419 knockdown ameliorates NF-kappaB/ NLRP3 inflammasome-mediated inflammation in diabetic nephropathy. Cell Death Dis. 2017 Feb;8(2):e2583.

20 Cao Y, Xu R, Xu X, Zhou Y, Cui L, He X, Downregulation of IncRNA CASC2 by microRNA-21 increases the proliferation and migration of renal cell carcinoma cellsMol Med Rep2016 Jul141101925

21 Bartel DP. MicroRNAs: target recognition and regulatory functions. Cell. 2009;136(2):215-33.

22 Sethupathy P. The promise and challenge of therapeutic microRNA silencing in diabetes and metabolic diseases. Curr Diab Rep. 2016;16(6):52.

23 Wu J, Lu K, Zhu M, Xie X, Ding Y, Shao X, et al. miR-485 suppresses inflammation and proliferation of mesangial cells in an in vitro model of diabetic nephropathy by targeting NOX5. Biochem Biophys Res Commun. 2020 Jan 22;521(4):984-90.

24 Li M, Guo Q, Cai H, Wang H, Ma Z, Zhang X. miR-218 regulates diabetic nephropathy via targeting IKK-beta and modulating NK-kappaB-mediated inflammation. J Cell Physiol. 2020 Apr;235(4):3362-71.

25 Bahudhanapati H, Tan J, Dutta JA, Strock SB, Sembrat J, Àlvarez D, et al. MicroRNA-144-3p targets relaxin/ insulin-like family peptide receptor 1 (RXFP1) expression in lung fibroblasts from patients with idiopathic pulmonary fibrosis. J Biol Chem. 2019;294(13):5008-22.

26 Li H, Shi H, Gao M, Ma N, Sun R. Long non-coding RNA CASC2 improved acute lung injury by regulating miR144-3p/AQP1 axis to reduce lung epithelial cell apoptosis. Cell Biosci. 2018;8:15.

27 Xie W, Silvers R, Ouellette M, Wu Z, Lu Q, Li H, et al. A luciferase reporter gene system for high-throughput screening of $\gamma$-globin gene activators. Methods Mol Biol. 2016;1439:207-26.

28 Ignarski M, Islam R, Müller RU. Long non-coding RNAs in kidney disease. Int J Mol Sci. 2019;20(13):3276.

29 Leti F, Morrison E, DiStefano JK. Long noncoding RNAs in the pathogenesis of diabetic kidney disease: implications for novel therapeutic strategies. Per Med. 2017;14(3):271-8.

30 Li Y, Zheng LL, Huang DG, Cao H, Gao YH, Fan ZC. LNCRNA CDKN2B-AS1 regulates mesangial cell proliferation and extracellular matrix accumulation via miR-424-5p/HMGA2 axis. Biomed Pharmacother. 2020;121 109622.

31 Lu Z, Liu N, Wang F. Epigenetic regulations in diabetic nephropathy. J Diabetes Res. 2017;2017:7805058.

32 Badal SS, Wang Y, Long J, Corcoran DL, Chang BH, Truong LD, et al. miR-93 regulates Msk2-mediated chromatin remodelling in diabetic nephropathyNat Commun2016 Jun 28712076

33 Schrijvers BF, De Vriese AS, Flyvbjerg A. From hyperglycemia to diabetic kidney disease: the role of metabolic, hemodynamic, intracellular factors and growth factors/cytokinesEndocr Rev2004 Dec2569711010

34 Ponting CP, Oliver PL, Reik W. Evolution and functions of long noncoding RNAs. Cell. 2009;136(4):629-41.

35 Tian Y-J, Wang Y-H, Xiao A-J, Li P-L, Guo J, Wang T-J, et al. Long noncoding RNA SBF2-AS1 act as a ceRNA to modulate cell proliferation via binding with miR-188-5p in acute myeloid leukemia. Artif Cells Nanomed Biotechnol. 2019;47(1):1730-7.

$36 \mathrm{Xu}$ H, Zhou M, Cao Y, Zhang D, Han M, Gao X, et al. Genome-wide analysis of long noncoding RNAs, microRNAs, and mRNAs forming a competing endogenous RNA network in repeated implantation failure. Gene. 2019;720: 144056.

37 Kong X, Liu CX, Wang GD, Yang H, Yao XM, Hua Q, et al. LncRNA LEGLTBC functions as a ceRNA to antagonize the effects of miR-34a on the downregulation of SIRT1 in glucolipotoxicity-induced INS-1 beta cell oxidative stress and apoptosis. Oxid Med Cell Longev. 2019;2019:4010764.

38 Hu WY, Wei HY, Li KM, Wang RB, Xu XQ, Feng R. LINC00511 as a ceRNA promotes cell malignant behaviors and correlates with prognosis of hepatocellular carcinoma patients by modulating miR-195/EYA1 axis. Biomed Pharmacother. 2020;121:109642.

39 Yao T, Zha D, Gao P, Shui H, Wu X. MiR-874 alleviates renal injury and inflammatory response in diabetic nephropathy through targeting toll-like receptor-4. J Cell Physiol. 2018;234(1):871-9.

40 Ortiz-Munoz G, Lopez-Parra V, Lopez-Franco O, Fernandez-Vizarra P, Mallavia B, Flores C, et al. Suppressors of cytokine signaling abrogate diabetic nephropathy. J Am Soc Nephrol. 2010;21:763-72.

41 Bao NN, Kong DY, Zhu D, Hao LR. Influence of overexpression of SOCS2 on cells of DN rat. Asian Pac J Trop Med. 2015;8(7):583-9. 